\title{
La inclusión del estudiantado ciego en la escuela regular en Cuba
}

\section{The Inclusion of Blind Students in Regular School in Cuba}

Carmen Caridad Díaz Morales

Universidad de Ciencias Pedagógicas Enrique José Varona

Calle 108 No. 29F08, 11400

La Habana

carmencdm@ucpejv.edu.cu

Cuba

\section{Resumen:}

En el presente trabajo se ofrecen reflexiones teóricas relacionadas con el proceso de inclusión del estudiantado ciego a la escuela regular y la posibilidad de la escuela para dar respuesta a las necesidades y potencialidades. Se toman como punto de partida, los estudios teóricos realizados por la autora y sus experiencias prácticas. La temática de la inclusión es un principio rector del sistema educacional cubano, que cuenta con respaldo político y jurídico, aún es insuficiente su puesta en práctica en relación con el estudiantado ciego. La inclusión supone un modelo de escuela en el cual profesorado, alumnado, familia y comunidad participan y desarrollan un sentido de colectividad entre todos, tengan o no discapacidades. Para lograr que los requerimientos educativos en el proceso de inclusión cuenten con la máxima calidad es necesario continuar perfeccionándolos con el objetivo de la inclusión total de las personas ciegas a la sociedad. En este artículo se ofrecen algunas propuestas que pueden resultar útiles para facilitar este proceso en la escuela cubana.

Palabras clave: Inclusión; ciego; escuela regular; educación especial.

\begin{abstract}
:
This paper provides some considerations regarding the process of inclusion of blind students in regular school, as well as the possibilities school has to meet students' needs. The foundations of this work are the author's theoretical studies and practical experiences. Although inclusion is a leading principle of the Cuban system of education, supported by political and juridical provisions, it still needs to be addressed in schools in what concerns blind students. In this respect, inclusion presupposes a model in which teachers, students, family, and community engage and develop a principle of collectivity regardless of disabilities. In order to accomplish higher education quality standards of inclusion for the blind, it is necessary to continue improving the total inclusion of these students in society. This article provides several proposals that might be useful to ease this process for Cuban schools.
\end{abstract}

Keywords: Inclusion; blind; regular school; special education.

Recibido: $15 / 11 / 2018$

Aceptado: 13/06/2019

Publicado: 18/06/2019

| pág. 121 - 141 
La inclusión del estudiantado ciego en la escuela regular en Cuba

\section{| Introducción}

La Agenda Universal 2030 de las Naciones Unidas (CEPAL, 2016) muestra un ambicioso plan de acción a favor del bienestar del planeta y de las personas. Plantea, como parte del objetivo de la paz, propiciar sociedades pacíficas, justas e inclusivas para que el mundo sea mejor; un mundo justo, equitativo, tolerante, abierto $\mathrm{y}$ socialmente inclusivo, en el que se atiendan las necesidades de los más vulnerables. Esta Agenda refleja las necesidades de niños, niñas, adolescentes, jóvenes, de las personas con discapacidad, entre otros, con una educación de calidad.

Según la UNESCO (2011), la inclusión es un enfoque que responde positivamente a la diversidad de las personas y a las diferencias individuales, entendiendo que la diversidad no es un problema sino una oportunidad para el enriquecimiento de la sociedad, mediante la activa participación de sus miembros en las comunidades, en la vida familiar, en la educación, en el trabajo y en todos los procesos sociales y culturales.

Tradicionalmente, el concepto de inclusión educativa ha sido restringido a definiciones de alumnos con necesidades especiales pero ha evolucionado hasta la idea de que niños, adolescentes y jóvenes tienen derecho a una educación inclusiva, que implica equivalentes oportunidades de aprendizaje en diferentes tipos de escuelas sin que influyan sus antecedentes sociales, culturales o sus diferencias en las habilidades y capacidades.

El racismo, el sexismo, el clasismo, la homofobia, la discapacidad y la intimidación tienen una raíz común que es la intolerancia a la diferencia y el abuso de poder para crear y perpetuar desigualdades. Hacer que las escuelas sean más inclusivas puede ser un proceso difícil para el 
profesorado, porque supone hacer un cuestionamiento respecto a sus propias prácticas y actitudes discriminatorias.

El objetivo de la inclusión es brindar respuestas apropiadas al amplio espectro de necesidades de aprendizaje, tanto en entornos formales como no formales de la educación. La educación inclusiva no constituye un tema marginal, sino que trata sobre cómo integrar a ciertos estudiantes a la enseñanza convencional. Representa una perspectiva que debe servir para analizar cómo transformar los sistemas educativos y otros entornos de aprendizaje, con el fin de responder a la diversidad de los estudiantes. El propósito de la educación inclusiva es permitir que los maestros y estudiantes se sientan cómodos ante la diversidad y la perciban no como un problema, sino como un desafío y una oportunidad para enriquecer las formas de enseñar y aprender (UNESCO, 2005).

El concepto de educación inclusiva, en los últimos quince años ha evolucionado hacia una idea plural. Considera a todos, no obstante las diferencias culturales, sociales y de aprendizaje, en igualdad de oportunidades para los diversos tipos de escuelas. El centro de atención se sitúa en la creación de entornos inclusivos. En este sentido, se coincide con lo planteado en la Cuadragésima Conferencia Internacional de Educación, celebrada en Ginebra (CIE, 2008), en la cual se asevera que la eliminación de los obstáculos a la participación de todos los educandos en el aprendizaje constituye el núcleo del concepto de una educación inclusiva verdaderamente arraigada en los derechos.

La inclusión educativa insiste en el desarrollo de la escuela regular y no en la reorganización de la escuela especial. Para lograr el éxito de este proceso se necesita un personal bien preparado, que cuente con recursos materiales y apoyos especializados de acuerdo con las particularidades del proceso de inclusión que se esté materializando. Lo anterior, sin dejar de considerar el papel de las familias y del propio alumnado.

Borges y Orosco (2014) consideran que la inclusión implica continuar aunando esfuerzos entre todos; es coordinar acciones, no es envolvernos en términos que no se 
ajusten a una realidad educativa estigmatizante. Es acoger a todos los ciudadanos y proporcionarles el derecho inalienable de pertenecer a un grupo y a no ser excluido; es no separar, marginar o apartar a alguien de otras personas, sin segregarlos por pertenecer a una "minoría en medio del grupo de individuos que forman la mayoría" (p. 42).

En la sociedad cubana, el estado desarrolla políticas explícitas y efectivas dirigidas a beneficiar a todos por igual. Entre sus propósitos se da prioridad a la labor pedagógica, se enfatiza en valorar la diversidad humana y la aceptación de las diferencias individuales. Respaldado por la Constitución de la República de Cuba (2002), cuyo contenido básico se sustenta en la igualdad plena de todos los ciudadanos.

La inclusión escolar de personas ciegas en la enseñanza regular constituye un significativo avance en materia educacional. A partir del triunfo de la Revolución Cubana se ha garantizado el éxito en relación con la educación inclusiva. Sin embargo, aún existen dificultades por superar. Es por ello que el presente artículo tiene como objetivo reflexionar acerca del proceso de inclusión del estudiantado ciego en la escuela regular cubana, además hacer algunas consideraciones en la búsqueda de alternativas posibles.

\section{|Aspectos Teóricos}

La educación puede ser un factor de cohesión social siprocura transformar la diversidad en un factor positivo de entendimiento mutuo entre los individuos y los grupos humanos y al mismo tiempo evita ser un factor de exclusión social (López, 2008). La inclusión educativa es un concepto propio de la pedagogía. Plantea que la escuela debe ser capaz de incluir a todas las personas en el proceso educativo sin importar su condición, origen, raza, religión, género 0 discapacidad.

García (2008) plantea que:

cuando nos referimos a personas con discapacidad, estamos vinculándonos a una persona con características propias que al mismo tiempo presenta características similares a 
las de las personas comunes, ... son personas como otras cualesquiera, pero con determinadas peculiaridades que las distinguen (p.18).

El estudiantado ciego puede ser objeto de exclusión social debido a la situación que se genera por su afectación visual. Predomina alrededor de estas personas conductas de sobreprotección y lástima que limitan su integración social, así como su pleno desarrollo.

Cuando tenemos ante nosotros un niño ciego, como objeto de educación, hay que trabajar no tanto con la ceguera, como con los conflictos que surgen en este niño al incorporarse a la vida. Por eso, la educación del niño deficiente no es más que la educación social (Vigotski, 1989, p.162).

El sistema sensorial visual proporciona una gama de conocimientos, resultado de la integración sensorial. Se relaciona con características de objetos y fenómenos de la realidad objetiva. Se reconoce que más del $80 \%$ del total de la información que se recibe es por la vía visual, mientras la audición garantiza el
$11 \%$ y el tacto, olfato y gusto que garantizan el 6\% restante (Castro Alegret, 2006). Este sistema se puede reestructurar mediante mecanismos de compensación garantizados por los procesos cognitivos, el lenguaje, la motricidad y otros.

Las acciones perceptivas no solo reflejan la situación presente, sino también anticipan, en alguna medida, las modificaciones que tienen lugar como resultado de las acciones prácticas. Al encontrarse afectadas las percepciones visuales en la persona ciega, resulta difícil que se pueda dar la anticipación de las acciones perceptivas para que se pueda regular la conducta de acuerdo con las condiciones y tareas que deberá enfrentar.

La visión permite una percepción simultánea de las cualidades de los objetos. Este proceso es más rápido, preciso y amplio por la vía visual que por la táctil. Esta última es una percepción sucesiva que puede afectar la integridad de las percepciones si no se le da el tratamiento adecuado. La ausencia de visión repercute en la formación de las representaciones del individuo ciego. Es un proceso 
que transcurre de forma más lenta porque no le resulta fácil integrar las imágenes sensoriales, que se están formando, para que pasen a escala racional. La organización sensorial que predomina en el caso de la ceguera es la táctil auditivo cinestésico.

Estudios realizados afirman que la restricción de información y experiencias reales de la persona ciega influye, de forma negativa, en todas las esferas de su formación. Sin embargo, necesita construir sus conocimientos, al igual que los videntes, en interacción directa con el medio físico, social y familiar que le rodea. Ello resulta palpable cuando se analiza el desarrollo del pensamiento operatorio concreto de las personas ciegas, el cual se debe producir con muchas nociones que implican componentes espaciales y manipular objetos con más lentitud que los videntes.

Resultados de investigaciones (Preisler, 1991; Rowland, 1983, 1984; Scott, 1969; Hernández, 2011; Díaz, 2006, 2012;) corroboran que el desarrollo social de los niños ciegos se ve afectado por la falta de información sobre las situaciones y relaciones sociales que proporciona la vista y da como resultado un acentuado egocentrismo y una pobre imagen de sí mismos, además de dificultades para compartir significados con otros.

Las limitaciones en la referida esfera social repercuten también en la adquisición y desarrollo de la comunicación, en el establecimiento de vínculos afectivos sólidos, dados por la escasa capacidad para establecer contacto social y en la esfera psicosexual. En la comunicación se puede evidenciar el llamado verbalismo. La persona ciega emplea palabras, frases 0 expresiones que le resultan carentes de significado porque su contenido es puramente visual; es decir, utilizan palabras de las cuales no poseen un referente perceptivo concreto.

La poca preparación que, en materia de sexualidad, aqueja a muchas personas ciegas, junto con la habitual ausencia de aptitudes para relacionarse socialmente, crea una propensión a los problemas de orden sexual. Al respecto Posse y Verdeguer (1991) aseguran que las personas ciegas desde el comienzo 
de sus relaciones interpersonales, ven limitadas de una $u$ otra forma la expresión de las necesidades sexuales propias de la edad, así como sus posibilidades para el amor y la vida de pareja porque tanto en el contexto familiar, escolar como social la educación sexual es insuficiente.

\section{Litvak afirma que:}

Las sensaciones y percepciones visuales no sólo revisten gran importancia para la cognición y la actividad práctica, sino también son fuentes inagotables de emociones estéticas, lo cual es propio, en especial, de la visión cromática, que contribuye a la mejor percepción de los objetos (1990, p.116).

En consecuencia, al estar afectado este sistema sensorial también se limita la imitación y el control de las expresiones emocionales.

La falta de visión repercute en la movilidad y la orientación espacial. Esto se puede manifestar en la asintonía de los movimientos y la limitación para trasladarse e incluso puede apreciarse hipotonía de los movimientos.
Se establece una gran diferencia entre las características perceptivas de la persona vidente y la ciega. En la primera, la percepción es global, parte del todo para recorrer las partes y se realiza principalmente por vía visual; en cambio, en la persona ciega la percepción es táctil-auditivaanalítica como vía fundamental. Por tanto, primero considera las distintas partes y a partir de ellas estructura un todo. La persona que carece de visión construye la realidad a partir de la información que le proporcionan los otros sistemas sensoriales, en unidad con la interacción social e instrumental.

Hay nociones que por ser exclusivamente visuales la persona ciega no logra aprender. Lo dicho anteriormente, de ningún modo significa que tenga un conocimiento "inferior" o "fragmentario", sino que conoce de forma diferente. Privilegia los sistemas sensoriales del tacto y el oído, utiliza el olfato y el gusto como sistemas de inmediatez.

Existe la creencia de que la persona ciega tiene "más desarrollados" los otros sistemas sensoriales. Esto no es así, la agudización de la percepción táctil, por ejemplo, es el resultado de la ejercitación y no de una compensación espontánea. 
Es necesario implementar una serie de cambios institucionales, curriculares y pedagógicos, de forma tal que la metodología orientada a los ritmos $y$ necesidades del estudiantado sea flexible y se ajuste a sus necesidades y características. Lograr esto conlleva un gran reto, tanto para las escuelas regulares, como para las escuelas especiales donde estudian niños, adolescentes y jóvenes con una discapacidad visual.

La inclusión implica reestructurar la política, la cultura y la práctica en las escuelas regulares y en las de enseñanza especial. Una escuela especial o una escuela general pueden o no ser escuelas inclusivas. Su condición de inclusiva está vinculada con la calidad de la educación que brindan. Por este motivo, es que se necesita dominar con profundidad las peculiaridades del aprendizaje de la persona ciega.

$\mathrm{Al}$ seguir los fundamentos del enfoque histórico-cultural (Vigotski, 1989), se considera a los factores sociales, a los mediadores $y$, especialmente, a los educativos como esenciales en la estimulación del desarrollo de esta persona ciega.
El profesorado de la enseñanza regular debe recibir preparación por parte de un especialista para identificarse con esa persona que va a incluir en su grupo. El diálogo entre ambos profesionales debe sustentarse en las fortalezas $y$ posibilidades de un sujeto que percibe el mundo privilegiando los sistemas sensoriales que no están dañados.

El profesorado de la enseñanza regular debe estar preparado para aceptar que el estudiantado ciego puede cumplir con el programa docente previsto, pero no utilizando los mismos métodos, procedimientos o recursos que se emplean con un vidente.

La educación tiene que concebirse como un proceso permanentemente en desarrollo, dinámico que sigue la marcha del progreso social, es por eso que se perfecciona $y$ modifica según las circunstancias lo demanden.

\section{|Una mirada a la educación especial en Cuba}

Todo cambio a nivel político conlleva transformaciones sociales que repercuten en la educación. 
Desde el triunfo de la Revolución Cubana en 1959 son significativos los cambios producidos en la educación. En Cuba, a diferencia de la mayoría de los países de América Latina, la educación es un derecho de todos y todas, es obligatoria, gratuita y estatal. Existen instituciones educativas hasta en los lugares más intrincados de la isla con la presencia de profesionales de la educación de todos los niveles educativos. El diseño curricular, de cada una de las educaciones es el mismo en todo el país, al igual que las estructuras de dirección.

La estructura del Sistema Nacional de Educación de la República de Cuba se instituye en niveles educativos que conforman una unidad: Primera Infancia, Educación Primaria, Educación Media Básica (Secundaria Básica), Educación Media Superior (Preuniversitaria), Educación Técnica y Profesional, Educación de Adultos, Educación Superior y Educación Especial. La revolución cubana significó un cambio en el modo de pensar sobre las personas con alguna discapacidad y los apoyos que se le podían proporcionar.
En el 1962 se crea el Subsistema Cubano de Educación Especial, dentro del sistema nacional de educación. Con el transcurso de los años se ha venido perfeccionando, al ritmo del desarrollo científico, técnico nacional e internacional.

Coincidiendo con Borges y Orosco (2014) la

Educación Especial es, una forma de enseñar, enriquecida por el empleo de todos los recursos necesarios, los apoyos, la creatividad que cada caso requiera $\mathrm{y}$ enriquecedora porque conduce y transforma, desarrolla al ritmo peculiar de cada alumno, pero de manera permanente y optimiza sus posibilidades (p. 4).

Este subsistema educativo presenta características similares al de los otros niveles educativos de Cuba. Para ello se implementa el mismo plan de estudios, pero se apoya en la creación y adecuación de programas especiales que permitan atender las necesidades del desarrollo del niño y su familia.

Actualmente, en el ámbito internacional, cuando se hace referencia a la Educación Especial 
se aboga por la eliminación de las escuelas especiales por ser consideradas "segregacionistas"; es decir, se defienden criterios de inclusión en la denominada nueva era de la integración.

La educación especial cubana tiene rasgos muy particulares y continúa defendiendo la existencia de la escuela especial porque los principios que la rigen no se contradicen con los preceptos de la inclusión. Se divide en dos grupos: las de carácter transitorio y las específicas. En las escuelas de carácter transitorio el estudiantado recibe preparación y apoyo en la formación de habilidades, las cuales asegurarán transitar a la enseñanza regular en el tiempo requerido para esos logros.

A las escuelas específicas asisten niños con diagnóstico de Retraso Mental. Su formación los sitúa para un egreso competente y los prepara para la vida adulta e independiente. Sobre todo con una formación laboral en correspondencia con sus posibilidades personales reales.

Múltiples argumentos evidencian la eficacia de las escuelas especiales en el contexto cubano. Ante todo porque el proyecto social cubano equipara la igualdad de oportunidades $\mathrm{y}$ derechos de todas las personas. No es restrictivo, todo lo contrario, se distingue por el estrecho vínculo entre el estudiantado, profesorado, familia y comunidad. Todos tienen las mismas posibilidades, tanto en la escuela especial como en la escuela regular. Son partícipes de todas las acciones curriculares y extracurriculares del sistema, evidenciado en los éxitos académicos y socio culturales, como concursos, olimpiadas deportivas, festivales culturales y otros.

En el caso particular de la escuela especial para niños con discapacidad visual, asisten niños con diferentes diagnósticos de afectación visual. La discapacidad visual se expresa en forma de ceguera o de baja visión. Solo nos referiremos a la ceguera. Se asume como definición de persona ciega la que ofrece la Organización Mundial de Salud (OMS): Persona que no tiene percepción de luz en ambos ojos o presenta un residuo visual útil para la movilidad, que puede ser visión cuenta dedos, percepción de luz y bultos.

En este tipo de escuela se encuentran programas de estudios que incluyen disciplinas 
orientadas a su formación integral, entre ellas: Rehabilitación Visual, Escritura plana, Estenografía Braille, Orientación y movilidad, Computación, Ábaco, Preparación para la Vida, Talleres. Además, reciben la atención especializada de oftalmólogos, pediatras, enfermeras, profesores de música y de educación física, logopedas, psicólogos y psicopedagogos.

La atención educativa a las personas con discapacidades visuales está organizada desde muy temprana edad a partir de su confirmación diagnóstica. Las familias empiezan a recibir orientación especializada por parte de la escuela desde que se detecta la discapacidad se tienen en cuenta las posibilidades de cada niño.

La familia tiene el derecho de decidir a qué centro escolar asistirá su hijo. No obstante recibe orientación para que su hijo asista a la escuela especial de manera transitoria. Desde los primeros cursos el estudiantado recibirá las didácticas particulares por profesionales expertos. Posteriormente podrán transitar exitosamente a la enseñanza regular.
Según nuestra experiencia si la persona ciega asistiera primeramente a la enseñanza regular le sería casi imposible acceder al curriculum de estudio como consecuencia de un mediador no especializado, que no posee la preparación adecuada en la adquisición de la lectoescritura en sistema Braille, calcular con el método ábaco u orientación y movimiento en el espacio.

El sistema de educación cubano está en continuo perfeccionamiento y no deja de ser una preocupación las debilidades en la atención a la diversidad, en cómo diseñar los proyectos educativos institucionales, cómo preparar a los docentes y las familias, cómo generar vínculos institucionales para proporcionar más calidad al proceso de inclusión de la persona ciega a la enseñanza regular y en visibilizar el concepto de una escuela inclusiva.

\section{|Propuestas que facilitarían el proceso de inclusión de la persona ciega}

Las propuestas que a continuación se brindan se conciben en los marcos educativos de la escuela 
especial y de la escuela regular en cuanto a la preparación de sus profesionales, docentes, directivos, familia y comunidad en el proceso de inclusión.

Si se considera que la inclusión es un proceso, que lleva su tiempo y preparación, debe ser planificado convenientemente. Se coincide con el psicólogo Coll (1994), que define que enseñar es, en primer lugar, saber cómo aprenden mis alumnos y, en segundo lugar, buscar los métodos, procedimientos y medios para que aprendan mejor. Por lo tanto, todo el sistema educativo tiene que hacer una revisión interna e identificar fortalezas y debilidades en relación con el proceso de inclusión de la persona ciega, para brindar las orientaciones precisas en el establecimiento de óptimas condiciones educativas que favorezcan la real equiparación de oportunidades.

\section{Exploración de los diferentes contextos educativos}

La escuela regular y la escuela especial, dentro del ámbito de la educación cubana, mantienen vínculos de colaboración, orientación y ayuda mutua para el perfeccionamiento del sistema educativo. En la escuela regular, donde se efectúe el proceso de inclusión, hay que realizar una exploración por parte de los profesionales de la escuela especial. Para lograr esto se apoyarán en entrevistas grupales, encuestas $\mathrm{y}$ observaciones al centro. Estos instrumentos investigativos permitirán identificar el nivel de sensibilización y disposición que posee todo el claustro de profesores, familias y estudiantado, en relación con ese proceso. La indagación requiere precisar en los recursos de que dispone el profesorado para enseñar al discapacitado visual y de los materiales con que contaría el ciego para su aprendizaje como texto digital, en audio, o en Braille.

Con la exploración también se constatarán los conocimientos que posee el profesorado de la escuela regular en relación con la discapacidad visual. El análisis de los recursos humanos brinda la oportunidad de potenciar los aspectos positivos y trazar estrategias encaminadas a minimizar en todo lo posible lo negativo. 
Relaciones conjuntas entre los diferentes niveles educativos.

Deben planearse varias visitas del profesorado de la enseñanza general a la escuela especial para que observen cómo se desenvuelve el estudiantado ciego en actividades docentes, de informática, deportivas, pioneriles, recreativas. Así podrán comprender las vías y formas de proceder en el aprendizaje del estudiantado ciego, apreciarán sus logros y precisarán el trabajo con los medios de enseñanza que utilizan. También valorarán la importancia de utilizar el objeto real, acompañado del lenguaje como recurso que posibilita completar los rasgos y cualidades de los objetos o fenómenos que no pueden ser percibidos. Es recomendable que en alguna de estas visitas pueda participar el estudiantado vidente, para que aprecie los éxitos de sus pares y pueda indagar sobre esta educación.

\section{Particularidades del desarrollo}

El especialista de la escuela especial debe destacar la importancia del bastón para que la persona ciega se desplace y oriente con éxito. Cuando el estudiante ciego hace sus primeras visitas a la escuela, donde va a ser incluido, es imprescindible hacerle un recorrido por toda la instalación e ir describiendo todos los detalles que le van a ser imprescindibles para andar de manera independiente y segura. Las señales visuales que se encuentran por toda la escuela, indicando donde está el baño, la dirección, las aulas, etc., para la persona invidente no son significativas, por esto es necesario dedicar tiempo al reconocimiento por el ciego de cuántos pasos aproximadamente lo separan del baño cuando está en el aula, o cuántos escalones tiene que subir para llegar al segundo piso, etc.

\section{Exigencias didácticas}

También es necesario recalcar en lo diferente que resulta el sistema de lectura y escritura que utiliza la persona ciega, el Braille. Es una metodología aceptada internacionalmente. Está diseñada para poder ser explorada de forma táctil. Mediante la combinación de seis puntos a relieve se representa todo el alfabeto, signos de puntuación y matemáticos, los números, etc. Cuando el vidente 
aprecia como lee y escribe la persona ciega, se queda sorprendido y admirado ante el empleo de las manos para leer y la destreza con que lo realizan. Aunque el resultado de lo escrito parece mayor en braille, porque una página escrita en braille ocupa más del doble de una página escrita en tinta, es la misma información.

\section{La discapacidad una potencialidad}

El profesorado de la escuela regular tiene que sentir que la persona ciega va a ser un apoyo, una fuente de conocimiento a la que puede acudir siempre que lo necesite, a la que se le puede preguntar, cuando tenga duda en cómo hacer más comprensible el contenido que se le va a impartir o qué adaptación pudiera ser la más asequible. Por ejemplo, en su mayoría, el profesorado de la enseñanza regular no domina la lectura en Braille, por lo que revisar una tarea escrita por la persona ciega va a resultar casi imposible, por este motivo deberá pedirle al propio estudiante ciego que le lea la tarea en alta voz e incluso le puede preguntar sobre la ortografía de alguna palabra en particular, para así corroborar si la escribió correctamente o no.

\section{La entrega pedagógica}

La entrega pedagógica va a ser otro momento de profundización e intercambio entre especialistas de ambas escuelas. Es el momento de detallar la caracterización psicopedagógica individual del estudiantado y con ella trazar las estrategias de intervención futuras. Será el espacio para tratar las particularidades individuales de la persona incluida, su área afectiva y cognoscitiva, las características de la comunidad de donde proviene; así como detallar las peculiaridades de la familia.

Vías de preparación de los diferentes recursos humanos

Es necesario fortalecer el trabajo metodológico con la creación de orientaciones metodológicas específicas, en las que se explique todo lo que se quiere lograr. Estas orientaciones deben ser flexibles, para que le posibiliten al profesorado de la enseñanza regular hacer transformaciones en función de las peculiaridades del estudiantado ciego. 
Otra sugerencia para lograr el proceso de inclusión de la persona ciega puede ser la posibilidad de compartir los espacios de preparación metodológica que desarrollan las direcciones municipales de educación. Si se está hablando de inclusión, no debe realizarse una preparación atomizada de los diferentes niveles de enseñanza si todas tienen como objetivo común la inclusión de todos.

Los cursos de superación constituyen otra vía para preparar al personal docente de todos los centros educacionales, en función de fortalecer sus conocimientos y habilidades para asumir adecuadamente el proceso de la inclusión. Estos pueden ser un espacio muy provechoso para el profesorado de la escuela regular. Deben tener una adecuada planificación y selección de los contenidos que se van a trabajar en los cuales se profundicen aspectos teóricos, prácticos y se vivencien las conquistas a lograr en las personas ciegas.

\section{Compartir espacios profesionales ciegos}

En Cuba se cuenta con profesionales ciegos dignos de admiración, por los éxitos que han alcanzado en lo profesional y personal. Contar con la presencia de alguno de ellos en intercambios, donde se haga referencia a sus potencialidades, resultaría muy provechoso para los videntes y para la persona ciega. El profesorado de la enseñanza regular va a comprobar que con una adecuada educación inclusiva la discapacidad visual no es una limitante para lograr la inclusión social. Para el invidente invitado también resultaría de mucha satisfacción poder transmitir sus experiencias. Además, será reconocido socialmente. Junto a la historia de éxito de cada persona ciega está un gran número de profesionales de la educación, una familia potenciadora y una voluntad individual digna de imitar.

\section{Vínculo con la familia}

Otra de las acciones que esta investigadora considera de vital importancia para el éxito de la inclusión del ciego a la enseñanza regular, es materializar charlas entre las familias de la escuela especial y las familias del estudiantado donde va a realizar el proceso de inclusión. Estos serán espacios que favorecerán 
la eliminación de mitos vinculados con las personas ciegas, elevarán el compromiso y sensibilidad de las familias para apoyar la labor de integración.

\section{Educación de la sexualidad}

Reforzar la labor de educación de la sexualidad desde las primeras edades, como parte de la formación integral de la personalidad del estudiantado ciego, es otra de las acciones a implementarse en el proceso de inclusión a la escuela regular. La sexualidad es una importante esfera de la personalidad que hay que atender. La familia y el profesorado deben promover de manera intencional las acciones que se realicen con el estudiantado. Es deber de la escuela educar la sexualidad, a la vez que es un derecho de la niñez y la juventud recibirla para tener la oportunidad de vivir espacios pedagógicos que les permitan estructurar sus valores, conocimientos, actitudes y habilidades que redundarán en favorecer la salud y elevar la calidad de vida.

Se deben también fortalecer los lazos de amistad entre personas ciegas y videntes. Siempre que sea posible, apoyar la presentación del estudiantado del aula donde está incluida la persona ciega con la invitación a palpar el rostro de sus nuevos compañeros. Este procedimiento es oportuno para el conocimiento de su descripción física.

\section{Apoyo de los medios de comunicación}

La tarea de los medios de comunicación es esencial. Les corresponde un papel protagónico, al tener el encargo social de sensibilizar y movilizar a la sociedad hacia la comprensión y necesidad de una educación inclusiva. Los mensajes educativos a través de spots televisivos son de mucha utilidad para llegar a la población de manera atractiva y precisa.

\section{Formación del profesional para la educación infantil en el nivel medio superior y universitario}

Resulta inminente, como parte de las acciones para lograr el proceso de inclusión, continuar perfeccionando los planes de estudio de la carrera de Educación Infantil en función de alcanzar un proyecto coherente en 
la conformación de planes de estudio y programas. En las asignaturas del currículo base de las carreras pedagógicas, se necesita enfatizar en cómo llevar a vías de hecho el tema de la inclusión. El estudiantado, desde la formación, debe diseñar actividades pedagógicas de inclusión dirigidas a especificidades generales de todo el estudiantado y no únicamente de aquellos que tienen alguna discapacidad.

\section{Orientación y seguimiento del estudiantado egresado}

La escuela regular y la escuela especial deben realizar labor de orientación y seguimiento a sus egresados, así como al profesorado que está asumiendo su formación. Se deben realizar con sistematicidad visitas de ayuda a la escuela regular, donde se intercambie con profesores y estudiantes. Esto permitirá saber los niveles de satisfacción que tienen ambas partes en el proceso de inclusión y si es necesario reformular alguna nueva acción dirigida a perfeccionar el trabajo que se está realizando. Ambos centros tienen que sentir que son un equipo de trabajo dirigido a lograr un mismo objetivo, la formación integral de la persona ciega.
Es esencial que el profesorado de la escuela regular interiorice, conscientemente, la esencialidad de su rol en la formación de la personalidad de los niños $o$ adolescentes ciegos. Tiene que estar convencido de que en la medida que su labor educativa sea de más calidad podrá ofrecer personas incluidas a la sociedad, capaces de crear, participar en la producción de bienes sociales, amar, formar su propia familia $\mathrm{y}$ proponerse metas como cualquier otro ciudadano.

\section{|Conclusiones}

La inclusión del estudiantado ciego a la escuela regular genera conocimientos y compromisos de todos los actores de la sociedad, desde su comprensión de un ser bio-psico-social que exige del conocimiento de sus particularidades psicopedagógicas como de las especificidades curriculares y la gestión del vínculo entre las distintas instituciones sociales y educativas.

Las particularidades del desarrollo del estudiantado ciego no son obstáculos para la obtención de éxitos en todos los proyectos de vida que se propongan, tanto en lo 
personal como lo institucional. Para lograr esto se necesita del compromiso y actuación de la sociedad a favor de la inclusión de todos. Su éxito dependerá, tanto de la preparación que tenga el profesorado de la escuela regular como el de la escuela especial; así como, del amor y respeto que muestren por toda la diversidad de estudiantes y familias con las que interactúen.
Debe ser responsabilidad y compromiso de los gobiernos e instituciones educativas, llevar a cabo iniciativas a favor de la inclusión educativa. Para lograr este propósito es necesario enfrentar con optimismo todos los desafíos que conllevan la educación y el desarrollo multifacético del estudiantado ciego. 


\section{|Referencias}

Borges, S. \& Orosco, M. (2014). Inclusión educativa y educación especial. Un horizonte singular y diverso para igualar las oportunidades de desarrollo. La Habana, Cuba: Ministerio de Educación.

CEPAL (2016). Agenda Universal 2030 y los objetivos de desarrollo sostenible. Una oportunidad para América Latina y el Caribe. Santiago, Chile: Naciones Unidas. Recuperado de http://www.sela.org/media/2262361/ agenda-2030-y-los-objetivos-de-desarrollo-sostenible.pdf

CIE (2008). La educación inclusiva: el camino del futuro: un desafío para compartir. 48 ${ }^{a}$ Reunión de la Conferencia Internacional de Educación. Ginebra, Suiza: Centro Internacional de Conferencias. Recuperado de https://bit.ly/2LsKoSC

Castro Alegret, P. L. (Ed.) (2006). El maestro ante la atención de la sexualidad del alumno diferente. La Habana, Cuba: Editorial Pueblo y Educación.

Coll, C. (1994). Fundamentos de la nueva reforma educativa. Conferencia impartida en curso. Madrid, España.

Constitución de la República de Cuba. (2002). La Habana, Cuba: Ediciones Políticas.

Díaz, C. (2006). Educación de la sexualidad de adolescentes ciegos. Elementos para reflexionar. Ponencia al Congreso Iberoamericano de Medicina y Salud Escolar y Universitaria. La Habana, Cuba: Ministerio de Salud Pública.

Díaz, C. (2012). Estrategia pedagógica para la educación de la sexualidad de adolescentes ciegos. Tesis en opción al Grado Científico de Doctor en Ciencias Pedagógicas. La Habana, Cuba: Universidad de Ciencias Pedagógicas “Enrique José Varona”.

García, M. (2008). Personas con discapacidad. ¿Iguales o diferentes? La Habana, Cuba: Editorial Félix Varela. 
Hernández, C. (2011). Desarrollo de las concepciones educativas de las personas con discapacidad visual. La Habana, Cuba: Editorial Pueblo y Educación.

Litvak, A. G. (1990). Tiflopsicología. Moscú, URSS: Editora Vneshtorgizdat.

López, N. (2008).Equidad educativa y desigualdad social. Desafíos de la educación en el nuevo escenario. Buenos Aires: IIP-UNESCO. Recuperado de http://unesdoc.unesco.org/images/0014/001425/142599s.pdf

Organización de las Naciones Unidas para la Educación, la Ciencia y la Cultura [UNESCO]. (2005). Equidad educativa y desigualdad social. Desafíos de la educación en el nuevo escenario social latinoamericano. Buenos Aires, Argentina. Recuperado de http://unesdoc.unesco.org/ images/0014/001425/142599s.pdf

Organización de las Naciones Unidas para la Educación, la Ciencia y la Cultura [UNESCO]. (2009). Directrices sobre políticas de inclusión en la educación. París, Francia. UNESCO. Recuperado de http://unesdoc.unesco.org/ images/0017/001778/177849s.pdf

Organización de las Naciones Unidas para la Educación, la Ciencia y la Cultura [UNESCO]. (2011). La Unesco y la Educación. Toda persona tiene derecho a la educación. Paris, Francia: UNESCO. Recuperado de http://unesdoc. unesco.org/images/0021/002127/212715s.pdf

Posse, F. \& Verdeguer, S. (1991). La sexualidad de las personas discapacitadas. Buenos Aires, Argentina: Editorial Fundación “Creando Espacios”.

Preisler, G. (1991). Early patterns of interaction between blind infants and their sighted mothers, En: ChildCare, Health and Developmet, 17(2), 65-90. Recuperado de http://dx.doi.org/10.1111/j.1365-2214.1991.tb00680.x

Rowland, C. (1983). Patterns of interaction between the blind and their mothers. En: A.E Mills (Eds.). Language acquisition in the blind: Normal and deficient. Londres, Inglaterra: Croom Helm. 
Rowland, C. (1984). Preverbal communication of blind infants and their mothers. En: Journal of visual Impairment and Blindness, 78(7), 297-302.

Scott, R. (1969). The socialization of blind children. En D. A. Goslin (Ed.). Handbook of Socialization Theory and Research. Chicago, IL: Rand McNally.

Vigotsky, L. S. (1989). Obras completas. La Habana, Cuba: Editorial Pueblo y Educación. 